\title{
COORDINATED REFORM AND LOCAL SOLUTIONS: TRANSFORMING THE UNIVERSITY SYSTEMS OF REWARD AND PROFESSIONAL ADVANCEMENT
}

\author{
Julie Risien, PhD. \\ Center for Research on Lifelong STEM Learning \\ Oregon State University \\ Roberta Nilson \\ Center for Research on Lifelong STEM Learning \\ Oregon State University
}

Contact: Julie.Risien@oregonstate.edu

JUNE 1, 2018

OREGON STATE UNIVERSITY

254 Gilbert Hall, Corvallis, OR 97331 



\section{Coordinated Reform and Local Solutions: Transforming the}

\section{University Systems of Reward and Professional Advancement}

Abstract: University systems of reward and professional advancement underlie many of the challenges faced by institutions of higher education. These systems present barriers for faculty and professional staff who wish to include public engagement in their practice. Similar barriers exist in other domains such as improving teaching, informing public policy, and developing industry partnerships. This essay synthesizes the status of these systems through review and analysis of existing literature, reports, and a series of focus groups conversations on engagement. We suggest a comprehensive approach that applies two critical actions to guide transformation: 1) coordination across the many domains seeking reform; and 2) learning from study of local processes and lived experiences of change in reward and advancement practices. We conclude such an approach needs a synchronizing organization that can bring together disparate efforts, make meaning of the complexity of this challenge, and chart a path forward.

Suggested Citation: Risien, J. \& Nilson, R. (2018). Coordinated Reform and Local Solutions: Transforming the University Systems of Reward and Professional Advancement. Corvallis, OR: Center for Research on Lifelong STEM Learning. 

...scholarship has to prove its worth not on its own

terms, but by service to the nation and the world.

Oscar Handlin, 1986

As public trust in institutions of higher education is eroding, universities struggle to demonstrate their value (Mishra, 2017) ${ }^{1}$. The systems of reward and professional advancement have failed to keep pace with the changing needs of society, and the changing face of the academic work force (O’Meara, 2011). In this essay, we aim to promote continued discourse about transforming university systems of rewards inclusive of, but not limited to, the policies and practices of promotion and tenure. We build on the influential work of Boyer and many others (2015) dedicated to a system of higher education that artfully prioritizes needs of society through a more complete and nuanced view of scholarship.

Similar to how others have argued for a systems approach to higher education reform (Fitzgerald et al., 2016), we emphasize the importance of this thinking for reward and advancement. We propose two critical actions to guide this transformation. First, a more coordinated effort across domains in need of transformation (i.e., across issues such as public engagement, use of evidence-based teaching practices, or efforts to broaden participation in science) is essential. Second, uncovering and broadly sharing local examples that demonstrate the fine-scale processes and practices of change are critical to understanding how to best support and guide reforms. Such information would provide a map to customize and propagate reform in a way that meets the needs of different disciplines and institutions as they adapt to shifting dynamics in academia and the university workforce.

The reflections herein emerged from recent efforts to examine how university systems and people do, and do not, support scientists who wish to conduct public engagement activities ${ }^{2}$. In considering the

\footnotetext{
${ }^{1}$ Public trust in the scientific community has remained stable since the 1970's as reported in Perceptions of Science in America (AAA\&S, 2018). Mishra (2017) comments on trust in universities as institutions.

${ }^{2}$ Here we use the term engagement in the broadest sense, as used in university discourse across disciplines, to describe an array of faculty and professional efforts to communicate with the public about scientific information or engage the public in scientific processes. The American Association for the Advancement of Science provides a
} 
role of these reward systems in the domain of public engagement, we find clarity about the immensity of the challenge. Any movement to shift reward structures for the benefit of a single domain may miss opportunity for more thoughtful and comprehensive reform. The system of reward and advancement are part of the culture and very fabric of universities and they seem impossible to change. However, there are examples of significant policy changes at the institutional scale. Perhaps even more important are smaller scale efforts, at the department or school level that thoughtfully and intentionally work to shift practices by developing position descriptions, conducting annual reviews, and assessing the contributions of both academic and para academic careers in ways that acknowledge and appreciate a broader conception of scholarship.

In the sections that follow, we offer background on the importance and challenges of public engagement in higher education. We do so through a summary of literature and reports that make recommendations about changing the reward systems to better support engagement. Additionally, we present evidence from a recent study that highlights how people experience the reward systems across levels of the university. Finally, we suggest a pathway for progress through coordinated reform designed and implemented based on learning from local successes. We refer to local throughout as a shorthand for work conducted at the department or school level. Local has less to do with the organizational structure and more with how disciplinary norms guide practices and govern unique realities of funding, teaching, engagement, and community or industry partnerships.

\section{BACKGROUND}

Public engagement is one way universities demonstrate the importance of research in the lives of the public and the value of higher education institutions in society. Such work is gaining importance as the perception that trust in science is eroding ${ }^{3}$ and the nation is experiencing what has been described as

robust aspirational definition of public engagement with science "intentional, meaningful interactions that provide opportunities for mutual learning between scientists and members of the public" (AAAS, 2016).

${ }^{3}$ Although this perception is frequently expressed in academia, a recent report from AAA\&S (2018) shows that public trust in science has remained relatively flat over the last decades. 
anti-science activism positioning scientists as somehow apart from society (Hockfield, 2018; Holt, 2018). In this section, we examine the known complexity of universities and challenges associated with systems of reward and professional advancement in the context of public engagement.

Several national scale indicators and developments suggest the landscape of university public engagement is shifting. The National Science Foundation's increasing commitment to broader impacts (NSF, 2014) may be the most explicit indicator of such changes but is only one example of expanding interest in the topic. The growing graduate student led organization Communicating Science, with an annual workshop known as ComSciCon, is another indicator. The student group aims to empower future leaders with science communication skills so they can effectively share the results from research with broad and diverse audiences. A significant development, that sparked this essay, was a 2018 series of learning workshops coordinated and supported by the Kavli, Gordon and Betty Moore, David and Lucile Packard, and Rita Allen Foundations ${ }^{4}$. The series was borne out of shared recognition that many scientists have appetite to engage with the public. The foundations recognized a robust research on science communication (Hilgard, 2017) and how people learn (NRC, 2000) as well as remarkable initiatives to support scientists' efforts ${ }^{5}$. They seized this opportune time to understand the landscape as a system and stimulate progress in the public engagement with the science domain. The learning workshops focused on how to support scientists in their communication and engagement efforts. These sessions were designed in response to the community's recognition that scientists often do not feel supported in their communication and engagement work. In addition, those who do support effective communication and engagement and may be isolated, limiting their capacity to learn from each other and elevate practice.

A dominant theme in discussions about improving university systems of reward and professional advancement arises during annual gatherings of several large organizations such as the Network of STEM

\footnotetext{
${ }^{4}$ The series of invitational learning workshops, Support Systems for Scientists Communication and Engagement: An Exploration of the People and Institutions Empowering Effective Impact, took place between December 2017 and May 2018 and have been archived here http://www.informalscience.org/support-systemsscientists-communication-and-engagement-exploration-people-and-institutions.

${ }^{5}$ Examples including AAAS Leshner Leadership Institute, Alan Alda Center for Communicating Science, Portal to the Public Network and COMPASS represent just a few of the many models and programs that exist.
} 
Education Centers, National Alliance for Broader Impacts, the American Association for the Advancement of Science. This theme also appears in several reports from the National Academies of Sciences, Engineering and Medicine, and many other venues.

We conducted a small study in preparation for the foundation-led learning workshops (see Risien and Nilson, 2018). This included a targeted review of recently published reports and articles, published within the last five years, to inform understanding of the current state of public engagement at universities. Our aim was to identify critiques and recommendations already recognized by the community to help workshop participants advance the conversation and avoid recreating existing sound recommendations for action.

To complement the literature review we also captured accounts of the lived experiences of individuals at various positions within universities through a series of seven video-conferenced focus groups; one each for graduate students, postdoctoral scholars, tenured faculty, facilitators and brokers of engagement, government and public relations, deans, and vice presidents or chancellors for research. Each group included three to five individuals, altogether representing 22 institutions; discussions lasted between 50 and 110 minutes. We designed the conversations to gather key insights about the lived experiences of scientists conducting engagement and others in supporting scientists' engagement efforts. Participants shared their engagement experiences then responded to a series of conversational prompts about 1) engagement supports and resources across levels at their institutions; 2) social and cultural aspects of engagement; 3) the roles of disciplinary societies and other external entities, mentorship, and training; and 4) university reward structures. Although we were unable to recruit department chairs for focus groups, we recognized that they have valuable insight on these topics. The study team alternatively scheduled informal conversations with three department chairs in very different disciplines. These conversations provided insights and an opportunity to check our assumption that chairs play a critical role of in reward and advancement. The interviews helped to build our understanding of the challenges and opportunities faced by department chairs. 


\section{THE STATE OF REWARD AND ADVANCEMENT FOR PUBLIC ENGAGEMENT}

Recommendations to change faculty incentive structures appear in reports and literature that span disciplines and topics (Fitzgerald et al., 2016; Hilgard, 2017; National Research Council, 2014; Neeley et al, 2014; O’Meara, Eatman, \& Peterson, 2015, among others). Furthermore, numerous publicly engaged scientists have shared personal examples of their engagement experiences and expressed concern with the lack of acknowledgement of these activities in job descriptions and career advancement decisions (Laurance, 2014; Lubchenco, 2017; Ngumbi, 2017; Sherman, 2013). Despite consistent recommendations to improve reward structures at universities, it is unclear if significant progress toward system-wide change is underway.

Many have demonstrated that inconsistent value is placed on public engagement across disciplines and universities (Christie et al., 2017; Sobrero \& Jayaratne, 2014). Our focus group discussions included personal examples demonstrative of this research and pointed to important differences in the perception of value of public engagement across faculty within the same department and discipline. For example, graduate student and post-doc conversations reflected on different attitudes towards public engagement among participants’ own faculty mentors as compared to faculty peers of the same institution and discipline. Faculty who were unsupportive of public engagement activities were likely to express sentiment that those activities were not important elements of being a scientist. Some scientists are not convinced that public engagement or science communication activities should be incentivized by institutions or funding agencies. In the words of one of these scientists captured by Kasumovic (2015):

Creating such incentives [to reward science communication] will result in funding poorer science and diluting of the quality of science communication. That is not what we need in a time when there seems to be a war on science... There is no doubt that science communication benefits the community, but it does not advance primary research. For that reason alone, recognizing anything that isn't primary research will jeopardize a program whose goal is to further such research. 
Heterogeneity of opinion on the value of public engagement within the scientific community points to unresolved issues. Outside the realm of those actively involved in practice and academic discussion around public engagement, the definition of public engagement remains murky. All focus groups discussed public engagement in terms of overcoming what they perceive as public knowledge deficits through communication or knowledge transfer. This observation aligns with recent research on how scientists prioritize public engagement objectives, which found that many scientists involved in public engagement hope to inform the public about science and defend science from misinformation, rather than establish trust and resonance with the public (Besley, Dudo, \& Yuan, 2017; Dudo \& Besley, 2016). Leaders and committees that oversee academic review and professional advancement are also not generally equipped to evaluate public engagement. The dominance of the deficit model, a relatively simplistic approach as compared to more engaged methods, may lead to challenges accounting for engagement quality and outcomes in processes of professional advancement. This has been also noted as a challenge in efforts to support more systemic engagement (McNall et al., 2015).

We observed that the rigidity of the promotion and tenure system favors maintenance of the status-quo implicitly excluding activities around public engagement at many universities. It is notable that, at least with regard to tenure-track faculty, those who participate in reviewing faculty for advancement were themselves successful in a system that does not account for engagement in any meaningful way. Leadership from offices of research acknowledged the real challenges and risks for pretenured faculty allocating efforts toward expanding and improving engagement. Deans explained that these risks breed cynicism and fatigue among faculty who "stick their necks out", to collaborate across disciplines and/or communicate with the public. All focus groups (and our conversations with department chairs) spoke of engagement as a set of activities piled on top of already overwhelming workloads. Deans and leadership from research offices shared their perception that the on-the-ground practice of assessing scientists is ultimately in the hands of department chairs and disciplinary peers. Deans also noted their own important role in supporting department chairs and a culture of engagement. Overall, the conversations with leadership demonstrated a lack of agency to effect change in the current system. 
Department chairs and faculty echoed concerns that academic peers sometimes perceive engagement activity as distasteful self-promotion. However, a recent national survey of U.S. scientists suggests that this attitude may be shifting as most scientists support the idea of taking an active role in public policy debates (Rainie, Funk, \& Anderson, 2015). Nevertheless, our focus groups reveal that universities still harbor a stigma around public engagement. Such experiences undoubtedly vary across disciplines. A minority of outspoken actors are likely the drivers of such negative perceptions of engagement. Still, we take these accounts as a signal that facets of academic culture are unsupportive and acting against the changes that would integrate engagement into the professional life of modern scientists.

It is important to note that there are vast differences between institutions, and within institutions colleges and departments, in terms of size, mission, decision making structures, level of teaching responsibility, scale of research activity, research applications, facilities, and degree to which the scientific work connects to industry or policy makers. These differences appear in other analysis that found significant disciplinary variation in reported degree and types of engagement across disciplines (Doberneck and Schweitzer, 2017). Even our conversations with three department chairs revealed vast differences in their perceptions of their own role in supporting public engagement. These ranged from no role, to aspirations to increase their level of support, to serving as an active facilitator and broker of engagement activity.

Focus group conversations with early-career faculty, graduate students, and post-docs highlighted how variation in departmental support for public engagement can function as a filter, encouraging many of the younger scientists most actively interested in engaging with the public to pursue careers outside of academia. Those who wish for public engagement to be a significant part of their professional lives are not confident that they will find supportive environments in academe. One focus group with professors discussed how some departments are "outright hostile” towards faculty who pursue public engagement work. The graduate students and post-docs that we spoke with were actively involved in public engagement and reported a variety of public engagement support from mentors. However, even those with the most supportive of mentors recognized the trait as unusual and noted that they had actively 
sought out mentors who valued engagement. These individuals expressed concern that the disregard for public engagement in job descriptions and promotion and tenure decisions dissuade them from pursuing university careers. As expressed by one graduate student:

People who are more likely to be the agents of change in the community, if they don't feel comfortable in that community, they are going to leave. So yeah sure, the people who are graduate students right now might have that inclination (to engage the public with their science), but that doesn't mean that is going to translate into the senior faculty level twenty or thirty years from now.

The participants of the graduate student focus group agreed: academe’s dominant processes of reward and professional advancement functions to prevent change in the system. They discussed how they and many of their peers are choosing to pursue a future outside of academia in favor of a career path that would provide more opportunity for public engagement. Furthermore, these early career scientists discussed how dismissive or stigmatized attitudes of senior faculty towards public engagement seeps into the minds of their peers. They expressed concern that such attitudes become internalized by the time those early career faculty are in positions to make decisions about reward and advancement. When asked specifically if they thought promotion and tenure was relevant to this discussion, another student explained:

I feel like that is locked in a safe, I'm never going to have a say ... I have no idea how to change that because the ones that control it are the people who have been in academia the longest, and those are going to be the hardest minds to change. I see that as one of the biggest problems, and I don't know how to fix it.

Some scholars have tried to address this barrier by providing recommendations to guide faculty on how to demonstrate the value of their public engagement work for consideration in promotion and tenure (Franz, 2009, 2011). Some universities are starting to utilize proprietary software to measure and evaluate the impacts of engagement. This practice has not been consistent across universities and it remains unclear whether all departments and disciplines will accept such documentation in processes of 
reward and advancement. Another component to the metrics and measurement challenge is the risks (perceived and real) associated with reliance on quantifiable metrics for measuring public engagement. In our conversation with deans, they noted that the most impactful work requires significant time and resources prior to observation of measurable results. They described the metrics and measures often used only track activity and numbers of participants, but fall short of indicators of quality, impact, or learning. Across focus groups, there was concern that the amount of time needed to form valuable community partnerships and programs that truly serve the community is woefully underestimated and undervalued. Some even spoke of a complete disregard for these efforts in processes of reward and advancement.

Review committees are generally ill-prepared to assess the quality of engagement activities as a consideration for advancement (McNall et al., 2015). However, there is notable progress in terms of institution-level policy that can offer top-down support for integration of engagement into review processes. For example, the Kellogg Commission on the future of Public and Land-grant Institutions (1996-2000) has resulted in updated promotion and tenure guidelines at many universities such that faculty involved in engagement activities can be recognized (Bryne, 2006). Often pointed to as an exemplar, Michigan State University integrated community engagement into institutional guidelines and explicitly embedding outreach and engagement into review forms. Arizona State University has opened up opportunity for mission-driven reforms, through their well-known transformation to become the New American University (Crow \& Dabars, 2015), which has made contributions to the public good and an educated democratic society explicitly part of ASU's mission. The assertions of leadership have paved the way for university-level culture change. However, individual departments have had varied responses in terms of changing practices associated with developing positions, hiring, review and advancement. These institutional policies are necessary for reform, but they are not sufficient to disrupt the culture that drives practice (Doberneck, 2016).

Despite all we know, we are still without a roadmap to guide comprehensive transformation of university systems of reward and advancement. Change makers may benefit from stepping back and considering what it really takes to disrupt the status quo and support transformation. Neither top-down not 
bottom-up approaches can alone drive transformation. It will take a more nuanced and intricate approach to shift structures and actions across disciplines, institutions, and domains in higher education. A first step is to embrace change as a complicated, contextual, ongoing, and non-linear process. Although we cannot make change happen, is can be guided through thoughtful and subtle shifts that, over time, improve conditions in the system to support the practices indicative of desired reforms (Archer, 2013; Chia, 1999, 2000, Kezar, 2014; Poole \& Van de Ven, 2004; Tsoukus \& Chia 2002).

\section{MAKING PROGRESS}

In our reflections about the status of university reward and advancement systems, we point to promotion and tenure in particular as a constraining condition that prevents progress on a variety of issues, from public engagement to improved education. There are efforts to change institutional policy, but very few policy changes affect everyday practice as long as implementation of such changes runs counter to cultural narratives about faculty excellence, defined primarily as research within one’s discipline. Siloed in specific domains, recommendations neglect to consider what could be gained from a more comprehensive and strategic approach. Our study did not find empirical accounts (in the literature or in focus groups) about how reward-related change in practice actually comes about, what occurs through the process of change, and how such changes may be improving public engagement outcomes.

We suggest a comprehensive and strategic approach to support systemic transformation in reward and advancement. We argue that pursuit of two paths, coordination across domains and learning from local efforts, may guide positive and effective reforms across scales. At the macro scale, we recommend a coordinated approach to understanding the role of university reward systems in making progress across domains. At the meso-scale, we believe there are critical lessons to learn from local scale changes already in progress. 


\section{Coordinated Reform}

Here we emphasize the role of reward systems in supporting public engagement. However, in suggesting an approach coordinated across domains, we expect that status of improvement in the public engagement domain would occur through transformation. Separate efforts may impede each other. On the other hand, there is promising potential for these efforts to work in concert, to learn from each other, and support mutually beneficial reforms. Often proposed as a solution for a single domain, reform is rarely consider across higher education. For example, Sanberg and others (2014) hone in on patents and commercialization, recommending that professional advancement be expanded beyond research publications, teaching, and service to explicitly include patents and commercialization activities. A recent report on the science of team science exposes promotion and tenure processes as one of the top five constraints on team-based science (NRC, 2015). Others considered promotion and tenure a major barrier to undergraduate STEM education reform (Weaver, Burgess, Childress, \& Slakey, 2015).

We posit that attempts to prioritize one reform over another may overwhelm our academic institutions' ability to adapt to and absorb change. Such competition may even invoke resistance, potentially enhance organizational rigidity as a response, and thus prevent further progress. One could imagine that attempts to include engagement explicitly in systems of reward be accomplished at the detriment of instructional reforms. Administration and faculty governance structures may have a limited tolerance for contentious reforms and be unwilling to tackle new efforts when previous ones (successful or not) consumed too much energy. The pace of reform may speed up and begin to address the backlog. Working together, leaders from across domains, could identify significant areas of alignment across their efforts, collaboratively address shared challenges, and work to prioritize efforts to across the system.

Such a strategic approach may find purchase and progress where the current disparate efforts cannot. However, it is important that a coordinated approach does not result in homogenous and inflexible systems of reward and advancement that could stymie innovations in engagement and other domains. There is no reasonable one-size-fits-all answer. Coordinated reform will need to provide a mechanism for systems to acknowledge the vast variety of work performed in the academy. Universities are places of 
creativity, learning, and discovery and any scaled effort to shift system of reward should elevate opportunity for experimentation and innovation. Additionally, a coordinated effort should establish mechanisms that enable learning across institutions, disciplines, and university domains.

\section{Learning from Local Success}

Despite the sentiment that promotion and tenure specifically, and reward structures more broadly are unchangeable, many local reforms are afoot. These department or school level efforts seek to better match position design, hiring, review, and advancement to the shifting professional landscape of universities. Closely tied to disciplinary norms, departments and their leadership generally possess the autonomy to alter the way they develop position descriptions and conduct hiring and review processes. Their work on the ground may be a gateway to broader transformation. Department chairs need the cover and support of college deans and upper administrations to be successful. However, their ability to push on the status quo through incremental and subtle means should not be underestimated.

Local leaders can be responsive to the many specific contexts and considerable variety across disciplines and locations. For instance, the societal role of a specific discipline may have shifted (e.g. genetics or robotics). The state policy and funding landscape may place unique demands on departmental faculty and professionals. Industry or community partnerships may be more, or less, essential in achieving locally desired impacts and applications of research. Local needs for teaching reforms may be disproportionately high to recover from a period of low-performance. These types of challenges are best addressed through finely tuned local scale actions, including adaptations to the practices associated with reward and advancement.

There are critical lessons to learn from local scale changes. In fact, coordinated change efforts would benefit grounding in research about the finer-scale processes that already support change at the local level. Others have begun to uncover this benefit in terms of collaborative and interdisciplinary work (Klein \& Falk-Krzesinski, 2017). We recommend that empirical study and comparative analysis of what supports and sustains change at the local level should be a priority. Expanding knowledge in this way can 
help to tie broader policy or structural changes at the university level with the lived experiences of academics and professionals in context.

Detailed case studies can help us understand the processes and mechanisms of reform at a resolution appropriate for local application. Such study can elucidate the answers to questions such as: How does local change emerge? What and who are the drivers of the process? What specific actions are critical to success? How does change in reward systems alter the professional experience for both champions and those more reluctant? What are the unintended consequences, both negative and positive, of different types of local reform? How have such changes impacted underrepresented and early career faculty?

Over time, researchers may be able to capture observable shifts in norms and practices along the cycle of developing position descriptions, hiring processes, annual reviews, and advancement decisions. Armed with this knowledge of how reform actually happens, we can better ascertain who wins and who loses, what improves and what may not. We can reveal the factors of success and uncover common challenges to guide future action and add thoughtful momentum to the transformation already in progress. Comprehensive case studies also complement the coordinated approach to broad reform. They could reveal how departments are accounting for collaboration, informing policy, alternative publication and creative contributions, direct and indirect societal impacts, broadening participation, and fostering partnerships to provide public services. Comparative analyses across cases would then reveal promising practices ripe for application in a variety of settings.

\section{CONCLUSION}

We have offered details about the status of university systems of reward and professional advancement in the context of supporting scientists who wish to conduct public engagement. We acknowledge that they must do so in the context of disciplinary norms of practice and institutional systems that may support or limit their success. We observed that agency to make change is low and restricted by a deeply embedded culture that regulates practices. The status quo constrains the degree to 
which universities, individuals, mentors, and groups pursue public engagement activities. There are however, signs of positive change.

The challenges presented by the systems of reward and advancement are vexing even outside the realm of public engagement, but also in many other domains such as transdisciplinary collaboration and improving teaching practice. Systems of promotion and tenure are often characterized, even among champions of reform, as too difficult to change. We suggest, though, that it is time for significant and comprehensive reform so that institutions of higher education better reflect the changing academic workforce and address the changing needs of society.

It is incumbent on the academy to shift the conversation, to reframe reform as a required strategic action that is already underway, but in need of thoughtful guidance. This process requires a coordinated approach and grounding in fine-resolution evidence about how practices are actually changed. Thoughtful attention to, and pursuit of, coordinated reform (at the macro scale) and learning from local changes (at the meso-scale) can strengthen systems of reward and professional advancement. Stronger systems would support a new university workforce with more diverse skills, a broader variety of professional roles, and creative and innovative solutions to society's most intractable challenges.

Those at the forefront of testing and reforming university systems of reward are taking noteworthy risks that could include job loss. High-level leadership must provide cover for those pushing to improve the system. While the university systems of reward are flawed, we must not completely reinvent them. There are significant protections and benefits in the current system that enable discovery and discourage normative science seeking specific and more desirable results. Transformation does not suggest trading our current system out for a new one, but rather evolving it to match universities as they aspire to be.

Coordinated reforms and thoughtful use of lessons from local efforts will require a wellrespected, knowledgeable and neutral convener to bring together disparate efforts across domains. Such an entity will need to make meaning in the context of conflict and complexity to map out a path of action. The approach recommended in this essay may help guide a scaled and thoughtful effort to address 
comprehensive reform. Universities are massive complex organizations with intricate culture. Many aspects of the academic universe are in need of change, but university systems of reward and professional advancement underlie all others. It is a regulator of diversity, teaching, mentorship, engagement, technology development, the future of our workforce and a democratic society. It is time to move reform of reward and professional advancement out of the shadows and into the light, exposing the strengths and flaws, to guide us to thoughtful transformation.

Acknowledgements: The authors wish to acknowledge the generous support of the Rita Allen Foundation that made this project possible and the Kavli Foundation for their unwavering bias toward action and systemic approaches that enhance the connection of science and society. Thank you our collaborative colleagues at the Center for Research on Lifelong STEM Learning. We also thank Ira Bennett and Barbara Natalizio for reviewing early drafts of this paper. 


\section{REFERENCES}

American Academy of Arts \& Sciences (AAA\&S). (2018). Perceptions of Science in America: A report from the Public Face of Science Initiative. Washington, D.C.: AAA\&S.

American Association for the Advancement of Science (AAAS). (2016). Logic Model for Public Engagement with Science. Washington, D.C.: AAAS.

Archer, M.S. (2013). Social origins of educational systems. London, U.K.: Routledge.

Besley, J.C., Dudo, A., \& Yuan, S. (2017). Scientists’ views about communication objectives. Public Understanding of Science, 00(0), 1-23.

Boyer, E. L., Moser, D., Ream, T. C., \& Braxton, J. M. (2015). Scholarship Reconsidered: Priorities of the Professoriate. San Fransisco, CA: Jossey-Bass.

Byrne, J.V. (2006). Public Higher Education Reform Five Years after the Kellogg Commission on the Future of State and Land-Grant Universities. Washington, D.C.: National Association of State Universities and Land-Grant Colleges.

Chia, R. (1999). A rhizomic model of organizational change and transformation: Perspective from a metaphysic of change. Journal of Management Studies, 10, 209-227.

Chia, R. (2014). Reflections: In Praise of Silent Transformation - Allowing Change Through 'Letting it Happen’. Journal of Change Management. Vol. 14, No. 1, 8-27.

Christie, L.D., Djupe, P.A., O’Rourke, S.P., \& Smith, E.S. (2017). Whose Job Is It, Anyway? The Place of Public Engagement in the Liberal Arts College. Journal of Higher Education Outreach and Engagement, 21(4), 23-50.

Crow, M.M., \& Dabars, W. B. (2015). Designing the New American University. JHU Press.

Doberneck, D.M. (2016). Are We There Yet?: Outreach and Engagement in the Consortium for Institutional Cooperation Promotion and Tenure Policies. Journal of Community Engagement and Scholarship, 9(1), 8-18.

Doberneck, D. M., \& Schweitzer, J. H. (2017). Disciplinary Variations in Publicly Engaged 
Scholarship: An Analysis Using the Biglan. Journal of Higher Education Outreach and Engagement, 21(1), 78-103.

Dudo, A., \& Besley, J. C. (2016). Scientists’ Prioritization of Communication Objectives for Public Engagement. PLOS ONE, 11(2), e0148867.

Fitzgerald, H. E., Bruns, K., Sonka, S. T., Furco, A., \& Swanson, L. (2016). The Centrality of Engagement in Higher Education: Reflections and Future Directions. Journal of Higher Education Outreach and Engagement, 20(1), 245-254.

Franz, N. (2009). A Holistic Model of Engaged Scholarship: Telling the Story across Higher Education's Missions. Journal of Higher Education Outreach and Engagement, 13(4), 31-51.

Franz, N. K. (2011). Tips for Constructing a Promotion and Tenure Dossier that Documents Engaged Scholarship Endeavors. Journal of Higher Education Outreach and Engagement, 15(3), 15-31.

Glass, C. R., Doberneck, D. M., \& Schweitzer, J. H. (2011). Unpacking Faculty Engagement: The Types of Activities Faculty Members Report as Publicly Engaged Scholarship During Promotion and Tenure. Journal of Higher Education Outreach and Engagement, 15(1), 7-31.

Handlin, O. (1986). Epilogue-Continuities. In B. Bailyn, D. Fleming, O. Handlin, \& S. Thernstrom (Eds.). Glimpses of the Harvard past. Caimbridge, MA: Harvard University Press, 129-131.

Hilgard, J. (2017). A Recap: Identifying and Overcoming Challenges to Science Featured in Attacks on Science. The Oxford Handbook of the Science of Science Communication.

Hockfield S. (2018). Our science, our society. Science 359(6375), 499.

Holt R. (2018). A tale of two cultures. Science 359(6374), 371.

Kasumovic, M. (2015). Science communication can be its own reward. Retrieved November 29, 2017, from http://theconversation.com/science-communication-can-be-its-own-reward-41747.

Kezar, A. (2013). How colleges change: Understanding, leading, and enacting change. New York, NY: Routledge. 
Klein, J.T., \& Falk-Krzesinski, H.J. (2017). Interdisciplinary and collaborative work: Framing promotion and tenure practices and policies. Research Policy, 46(6), 1055-1061.

Laurance, B. (2014). We need to stop punishing scientists for talking to the public. Retrieved November 29, 2017, from http://theconversation.com/we-need-to-stop-punishing-scientists-for-talking-to-the-

\section{public-41026.}

Lubchenco, J. (2017). Environmental science in a post-truth world. Frontiers in Ecology and the Environment, 15(1), 3-3.

McNall, M. A., Barnes-Najor, J. V., Brown, R. E., Doberneck, D. M., \& Fitzgerald, H. E. (2015). Systemic Engagement: Universities as Partners in Systemic Approaches to Community Change. Journal of Higher Education Outreach and Engagement, 19(1), 7-32.

Mishra, A. K. (2017). Restoring Trust In Higher Education: Making the Investment Worthwhile Again. Santa Barbara: ABC-CLIO, LLC.

National Research Council. (2000). How people learn: Brain, mind, experience, and school: Expanded edition. National Academies Press.

National Research Council. (2012). Research Universities and the Future of America: Ten Breakthrough Actions Vital to our Nation's Prosperity and Security. Committee on Research Universities. Board on Higher Education and Workforce. Policy and Global Affairs Washington, DC: The National Academies Press. Retrieved from http://www.nap.edu/catalog.php?record_id=13396 [May 2014].

National Research Council. (2014). Sustainable Infrastructures for Life Science Communication: Workshop Summary.

National Research Council. (2015). Enhancing the effectiveness of team science. Washington, D.C.: National Academies Press.

National Science Foundation. (2014). Perspectives on Broader Impacts. Washington, D.C.: NSF. Retrieved from http://www.nsf.gov/od/iia/publications/Broader_Impacts.pdf.

Neeley, L., Goldman, E., Smith, B., Baron, N., \& Sunu, S. (2014). GradSciComm Report and Recommendations: Mapping the Pathways to Integrate Science Communication Training into STEM 
Graduate Education | InformalScience.org. Retrieved from

https://www.informalscience.org/gradscicomm-report-and-recommendations-mapping-pathwaysintegrate-science-communication-training

Ngumbi, E. (2017). Scientists Should Talk Directly to the Public. Retrieved October 30, 2017, from https://blogs.scientificamerican.com/observations/scientists-should-talk-directly-to-the-public/

O’Meara, K. (2011). Rewarding multiple forms of scholarship: Promotion and tenure. In H. E. Fitzgerald, C. Burack, \& S. D. Seifer (Eds.), Handbook of engaged scholarship: Contemporary landscapes, future directions: Vol. 1. Institutional change (pp. 271-294). East Lansing, MI: Michigan State University Press.

O’Meara, K., Eatman, T. \& Peterson, S. (2015). Advancing Engaged Scholarship in Promotion and Tenure: A Roadmap and Call for Reform. Liberal Education, 101(3).

Poole, M. S., \& Ven, A. H. V. de. (2004). Handbook of Organizational Change and Innovation. New York: Oxford University Press.

Risien, J., \& Nilson, R. (2018). Landscape overview of university systems and people supporting scientists in their public engagement efforts: summary of existing recommendations and evidence from the field. Report to the Kavli, Rita Allen, Packard, and Moore Foundations. http://informalscience.org/support-systems-scientists-communication-and-engagement-explorationpeople-and-institutions.

Sanberg, P., Gharib, M., Harker, P., Kaler, E., Marchase, R., Sands, T., ...Sarkar, S. (2014). Changing the academic culture: Valuing patents and commercialization toward tenure and career advancement. Proceedings Of The National Academy Of Sciences, 111(18), 6542-6547.

Sherman, D. J. (2013). Partnering to Survive: Reflections on the Pursuit of Campus-Community Initiatives Prior to Tenure. Journal of Higher Education Outreach and Engagement, 17(4), 155-175. 
Sobrero, P., \& Jayaratne, K. S. U. (2014). Scholarship Perceptions of Academic Department Heads: Implications for Promoting Faculty Community Engagement Scholarship. Journal of Higher Education Outreach and Engagement, 18(1), 123-153.

Tsoukas, H., \& Chia, R. (2002) On Organizational Becoming: Rethinking Organizational Change. Organization Science 13(5):567-582.

Weaver, G. C., Burgess, W. D., Childress, A. L., \& Slakey, L. (Eds.). (2015). Transforming Institutions: 21st Century Undergraduate STEM Education. Purdue, IN; Purdue University Press. 